\title{
Auxin is a long-range signal that acts independently of ethylene signaling on leaf abscission in Populus
}

\author{
Xu Jin 1,2, Jorma Zimmermann't, Andrea Polle² and Urs Fischer ${ }^{1,2 *}$ \\ ${ }^{1}$ Department of Forest Genetics and Plant Physiology, Umeå Plant Science Centre, Swedish University of Agricultural \\ Sciences, Umeå, Sweden, ${ }^{2}$ Forest Botany and Tree Physiology, Georg-August University of Göttingen, Göttingen, Germany
}

\section{OPEN ACCESS}

Edited by:

Roberts Alan Jeremy,

University of Nottingham, UK

Reviewed by:

Shogo Matsumoto, Nagoya University, Japan

Cai-Zhong Jiang,

United States Department of Agriculture - Agricultural Research

Service, USA

*Correspondence: Urs Fischer,

Department of Forest Genetics and Plant Physiology, Umeå Plant Science Centre, Swedish University of Agricultural Sciences, SE-901 83

Umeå, Sweden urs.fischer@slu.se

tPresent address: Jorma Zimmermann,

Plant Ecology and Ecosystem Research, Georg-August University of Göttingen, DE-37073, Göttingen,

Germany

Specialty section:

This article was submitted to Crop Science and Horticulture, a section of the journal

Frontiers in Plant Science

Received: 01 April 2015

Accepted: 31 July 2015

Published: 12 August 2015

Citation:

Jin X, Zimmermann J, Polle A and Fischer $U$ (2015) Auxin is

a long-range signal that acts independently of ethylene signaling on

leaf abscission in Populus.

Front. Plant Sci. 6:634

doi: 10.3389/fp/s.2015.00634
Timing of leaf abscission is an important trait for biomass production and seasonal acclimation in deciduous trees. The signaling leading to organ separation, from the external cue (decreasing photoperiod) to ethylene-regulated hydrolysis of the middle lamellae in the abscission zone, is only poorly understood. Data from annual species indicate that the formation of an auxin gradient spanning the abscission zone regulates the timing of abscission. We established an experimental system in Populus to induce leaf shedding synchronously under controlled greenhouse conditions in order to test the function of auxin in leaf abscission. Here, we show that exogenous auxin delayed abscission of dark-induced leaves over short and long distances and that a new auxin response maximum preceded the formation of an abscission zone. Several auxin transporters were down-regulated during abscission and inhibition of polar auxin transport delayed leaf shedding. Ethylene signaling was not involved in the regulation of these auxin transporters and in the formation of an abscission zone, but was required for the expression of hydrolytic enzymes associated with cell separation. Since exogenous auxin delayed abscission in absence of ethylene signaling auxin likely acts independently of ethylene signaling on cell separation.

Keywords: Populus, auxin, ethylene, abscission, cell separation, PIN proteins

\section{Introduction}

In contrast to animal cells, most plant cells are tightly glued together by the middle lamella, such that even subtle positional changes to neighboring cells are impeded. During the life cycle of plants, however, many developmental processes require cell separation, e.g. anther dehiscence, floral organ abscission, pod shatter, radicle emergence and leaf abscission (Roberts et al., 2002; Lewis et al., 2006). Although the enzymatic activities required for the hydrolysis of middle lamellae and cell walls in various separation phenomena are thought to be similar, the triggers and therefore the upstream signaling are obviously diverse (Taylor and Whitelaw, 2001; Roberts et al., 2002; Fischer and Polle, 2010). While stress-induced and developmentally regulated organ abscission is relatively well studied little attention has been paid to the understanding of how seasonal cues are integrated to trigger the separation of organs from the plant body. In temperate climates the most remarkable cell separation process is the autumnal shedding of leaves from trees in fall. Despite of the paramount importance of leaf shedding as an adaptation to freezing and for nutrient cycling in forest ecosystems (Aponte et al., 2013; Zanne et al., 2014) our understanding of autumnal leaf abscission is limited. 
Important roles in timing of abscission have been assigned to the plant hormones ethylene and auxin. La Rue (1936) showed that removal of the leaf blade induces abscission; but when auxin is applied to the site of removal, abscission is inhibited. Addicott and Lynch (1955), Addicott et al. (1955) and Louie and Addicott (1970) applied auxin to decapitated stems of various annual species, which resulted in premature abscission of cotyledons or leaves. They found that not the absolute concentration of auxin but the ratio between distal and proximal auxin was relevant for the timing of abscission (Addicott et al., 1955; Louie and Addicott, 1970). Lower auxin concentrations on the distal than on the proximal side of the abscission zone favored abscission, whereas relatively higher auxin concentrations on the distal side delayed abscission (Louie and Addicott, 1970). From these experiments, it was concluded that an auxin gradient spans the abscission zone and regulates the induction of abscission (Addicott et al., 1955). Although testing of the auxin gradient model has been proven difficult in absence of highly resolved auxin concentration measurements or appropriate auxin response reporters the importance of auxin has further been strengthened by genetic evidence from the model plant Arabidopsis. Since petioles and pedicels Arabidopsis do not develop functional abscission zones (Patterson, 2001), research has focused on abscission of floral organs and dehiscence. Mutations in the auxin response factors $A R F 1$ and $A R F 2$ cause delayed petal abscission (Ellis et al., 2005). Similarly, expression of iaaL, which inactivates auxin, or AXR3-1, which is a stabilized Aux/IAA variant causing auxin resistance, under the control of an abscission zone specific promoter delays abscission (Basu et al., 2013).

Ethylene has been shown to play an antagonistic role to auxin in abscission of various organs. In the ethylene-insensitive Arabidopsis mutants ein2 and etr1-1 abscission is delayed (Bleecker and Patterson, 1997; Patterson and Bleecker, 2004), while application of ethylene hastens abscission in various organs and species. In line with a promotive role of ethylene in cell separation, ethylene levels often increase shortly before organ separation and ethylene is sufficient to induce the expression of a polygalacturonase required for cell separation in tomato petioles (Hong et al., 2000; Jiang et al., 2008). Interestingly, the same polygalacturonase is inhibited by the exogenous application of auxin, underlying the suggested antagonistic effects of auxin and ethylene in abscission (Hong et al., 2000). A broadly accepted model of hormonal interaction during organ separation suggests that a depletion of auxin levels in the abscission zone renders cells more sensitive to ethylene, which in turn induces secretion of middle lamellae hydrolyzing enzymes (Estornell et al., 2013).

Although the physiology and transcriptional changes related to autumnal leaf abscission in trees have been subject to intense studies (Chen et al., 1997; Keskitalo et al., 2005; Street et al., 2006; Giovannelli et al., 2007), mechanistic understanding of autumnal abscission signaling, especially the role of auxin, is limited. Here, we describe an experimental system to induce leaf abscission in Populus synchronously and report that auxin is a plausible long-range signal regulating abscission that acts independently of ethylene signaling.

\section{Materials and Methods}

\section{Plant Material and Growth Conditions}

PttPIN1b::GUS, PttPIN5b::GUS, PttWAT1::GUS constructs were transformed into hybrid aspen (Populus tremula L. $\mathrm{X}$ P. tremuloides Michx.; clone T89). The in vitro growth conditions were according to Love et al. (2009). Briefly, trees were grown in clear polypropylene containers (height, $14 \mathrm{~cm}$; diameter, $10 \mathrm{~cm}$ ) with OS140ODS140 gas-exchange spore filters (Combiness) and cultured on Murashige and Skoog medium (2.2 g. $\mathrm{l}^{-1}, \mathrm{pH}$ 5.6, Duchefa) with Phytagel P8169 solidifying agent $\left(2.7 \mathrm{~g} \cdot \mathrm{l}^{-1}\right.$; Sigma-Aldrich). Temperature was $22 / 18^{\circ} \mathrm{C}$ (light/dark), photoperiod was $16 \mathrm{~h}$, and light intensity was $90 \mu \mathrm{mol} \cdot \mathrm{m}^{-2} \cdot \mathrm{s}^{-1}$. The 3 to 4 -weeks-old in vitro grown transgenic trees (height, $10-12 \mathrm{~cm}$ ) were transferred in 2.51 pots with the commercial soil-sand-fertilizer mixture (Yrkes Plantjord Kronmull; Weibulls Horto, Hammenhög, Sweden) and grew in glasshouse under 18 -h photoperiod at $20^{\circ} \mathrm{C}: 15^{\circ} \mathrm{C}$ (light : dark). Trees were fertilized with $150 \mathrm{ml}$ of $1 \%$ Rika-S (N/P/K 7:1:5; Weibulls Horto) once a week (Vahala et al., 2013). The GH3::GUS lines are in the Populus $\times$ canescens backgroud, a hybrid between $P$. alba L. $\times$ P. tremula L. (Teichmann et al., 2008). These lines were used for the exogenous auxin and auxin transport inhibitor applications (Figures 2 and 4). All the other experiments were conducted in the T89 background.

\section{Dark Induction and Treatments with Auxin and Auxin Inhibitors}

For dark-induction experiments, fully expanded leaves with a petiolar angle of 75 to $90^{\circ}$ from 1.5 to $2 \mathrm{~m}$ tall trees were selected. Leaf blades were covered with aluminum bags under standard greenhouse conditions. Control samples were bagged in transparent plastic bags of the same weight. Each bag was labeled with a unique code referring to the genotype, tree replicate, leaf number, and treatment. Trees were gently shaken once per day, the dropped bags collected and the identifiers recorded.

Lanolin paste (Sigma-Aldrich) containing either $100 \mu \mathrm{M} \mathrm{IAA}$, 2, 4-D, or morphactin (CF, Sigma-Aldrich) were placed with the help of a disposable $100 \mu \mathrm{l}$ plastic tip at the junction between stem and petiole. For distal IAA application the lanolin paste containng $500 \mu \mathrm{M}$ IAA was applied to the junction between the petiole and leaf blade.

For auxin induction experiment (Supplementary Figure S1), in vitro grown young shoots with 5-6 leaves of the respective GUS lines were treated with auxin [1 $\mu \mathrm{M}$ 1-naphthaleneacetic acid (1NAA, Sigma-Aldrich) in $1 / 2$ MS-medium ( $\mathrm{pH} 5.8$, Duchefa)] for $3 \mathrm{~h}$ in darkness and washed with $1 / 2 \mathrm{MS}$-medium and assayed for GUS.

\section{Cloning of Promoter Sequences and Plant Transformation}

Nine hundred to thousand bp long promoter fragments of PttPIN1b, PttPIN5b, and PttWAT1 were PCR amplified from T89 using the primer combinations, PromPttPIN1b for/PromPttPIN1b rev, PromPttPIN5b for/PromPttPIN5b rev, or PromPttWAT1 for/PromPttWAT1 rev, respectively (Supplementary Table S3). The PCR products representing 
promoter regions of PttPIN1b, PttPIN5b, or PttWAT1 were subcloned into pGemTeasy and moved directionally as PstISalI, SalI-EcoRI, or HindIII-BamHI fragments into the vector pCambia1391Z (http://www.cambia.org), respectively. The resulting plasmids were transformed into Agrobacterium tumefaciens strain GV3101 (pMP90, pSoup) and used for stable transformations of the hybrid aspen clone T89 according to Nilsson et al. (1992). For all constructs at least five lines were selected and tested for GUS expression. Three lines each were analyzed in detail and representative GUS expression patterns are shown in Figure 5.

\section{Gene Expression Analyses}

Total RNA was extracted from 3-mm-long petioles near the junction to the stem using RNeasy Plant Mini Kit (Qiagen). The extracted total RNA was quantified with a ND-1000 NanoDrop spectrophotometer (NanoDrop Technologies, Wilmington, DE, USA). Two micrograms of total RNA was used as a template for reverse transcription with the QuantiTect Reverse Transcription Kit (Qiagen). Equal amounts of first-strand cDNAs were used as templates for real-time PCR amplification using the following primer combinations: qPttPIN1bforw/qPttPIN1brev; qPttPIN5bforw/qPttPIN5brev, and $q$ PttWAT1forw/qPttWAT1rev. The T89 actin gene, Actin1, was amplified using primer combination qPttActinlforw/ qPttActin1rev (Supplementary Table S3). Quantitative real-time PCR was performed using LightCycler ${ }^{\circledR} 480$ SYBR Green I Master (Roche Diagnostics GmbH, Mannheim, Germany) with a LC4800 (Roche Diagnostics) qPCR machine. PttPIN1b, PttPIN5b and PttWAT1 transcript levels were quantified in relation to Actin1 levels. Microarrays (61 k Affymetrix Poplar array) employing four biological replicates were performed by MFTServices (Tuebingen, Germany). Data analysis has been performed with the help of the Robin software package (Lohse et al., 2010).

\section{Histochemical Staining for GUS Activity}

GUS expression patterns were determined in 3-mm-thick longitudinal median sections of leaf axils. Samples were infiltrated employing vacuum for $30 \mathrm{~min}$ with GUS buffer (50 mM sodium phosphate buffer ( $\mathrm{pH} \mathrm{7.2),} 5 \mathrm{mM} \mathrm{K}_{3} \mathrm{Fe}(\mathrm{CN})_{6}$, $5 \mathrm{mM} \mathrm{K} \mathrm{K}_{4} \mathrm{Fe}(\mathrm{CN})_{6}, 0.1 \%$ Triton $\left.\mathrm{X}-100\right)$ containing $2 \mathrm{mM}$ 5-bromo-4-chloro-3-indolyl b-D-glucuronic acid (Duchefa Biochemie bv, The Netherlands) and then incubated in the dark at $37^{\circ} \mathrm{C}$ for $3-18 \mathrm{~h}$. Pigments were removed in $70 \%(\mathrm{v} / \mathrm{v})$ ethanol with gentle shaking. GUS expression patterns were examined with a bright-field microscope (Zeiss, Auxioplan) at low magnification $(\times 2.5, \times 10)$ or a scanner (Epson Perfection V600 Photo).

\section{Results}

\section{An Auxin Response Maximum Precedes the Formation of an Abscission Zone}

In Populus, gradual reduction of day length and temperature induces leaf abscission; whereas over-expression of phytochrome
$\mathrm{A}$ is sufficient to prevent abscission under these conditions (Olsen et al., 1997). However, it remains unclear if shorter day length is sensed by individual leaf blades, apices or if it is rather a response of the whole plant to reduced photoperiod. Therefore, we tested if shading of the leaf blade is sufficient to induce abscission in greenhouse-grown Populus trees. To this end we covered blades but not petioles of fully expanded leaves with aluminum foil bags. Shading induced cell divisions in the leaf axil and the formation of typical abscission zones 6 and 9 days, respectively, after the treatment started. Although the petioles were not shaded strong de-greening of the petiole was observed (Figure 1). By contrast, leaves in transparent bags of the same tree did not develop an abscission zone, were not shed and their petioles did not de-greened during the same period of observation (Figures 1A,E). Taken together, these results suggest that reduced photoperiod is sensed in the leaf blade and a mobile signal transports this information from the blade to the abscission zone, where it induces the development of an abscission zone.

Previous work in explants of annual species indicated that auxin could contribute to a leaf blade-derived abscission inhibiting signal (Louie and Addicott, 1970). We therefore

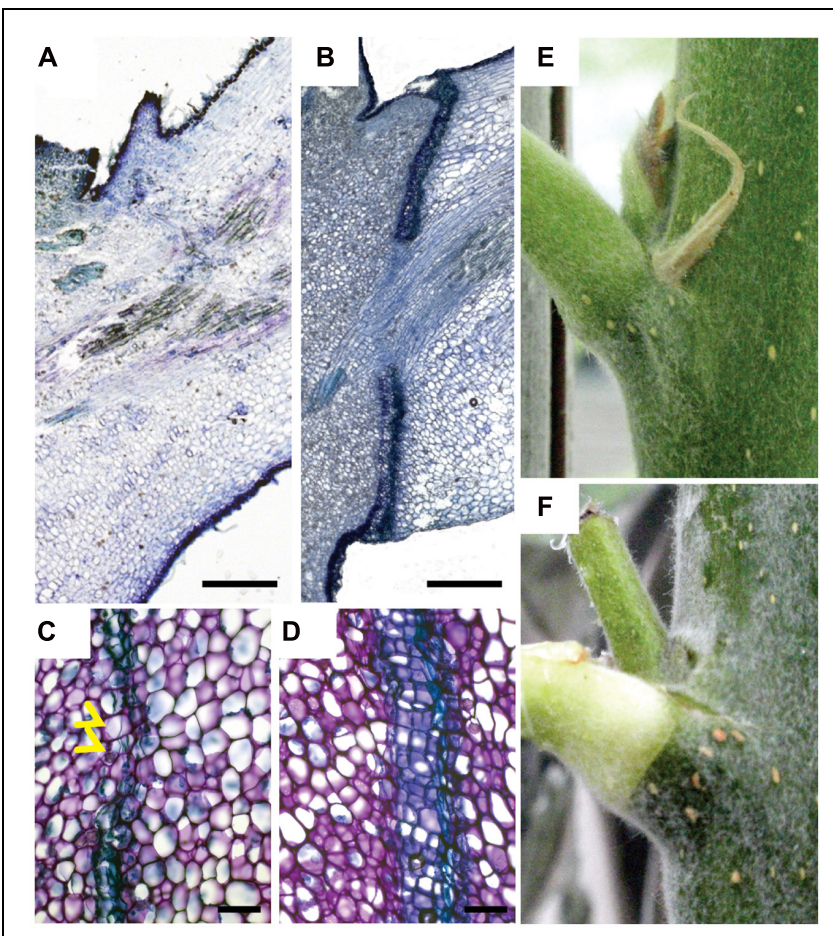

FIGURE 1 | A mobile signal from the leaf blade induces leaf abscission. (A-D) Toluidine blue stained longitudinal median section of leaf axils. (A) No abscission zone visible in axil of non-shaded leaf blade. Blade was bagged in a transparent plastic bag for 9 days. (B) Mature abscission zone in an axil of a shaded leaf blade. Blade was covered for 9 days in aluminum foil. (C,D) Abscission zones 6 and 9 days after shading started, respectively. Yellow arrowheads point to dividing cells. (E) Leaf axil of a non-shaded blade.

(F) Leaf axil of a blade shaded for 12 days. Chlorophyll distal to the abscission zone is degraded although leaf petiole was not shaded. Scale bars, $500 \mu \mathrm{m}$ (A,B); $100 \mu \mathrm{m}$ (C,D). 
examined the expression patterns of the auxin response reporter GH3::GUS (Teichmann et al., 2008). In control petioles, GH3::GUS was strongest in the vascular tissues (Figure 2A). After 6 days of shading, this activity became weaker but at the lower (abaxial) side of the petiole a new auxin response maximum emerged, which gradually expanded to the upper (adaxial, after 9 days) side of the petiole preceding the formation and maturation of the abscission zone (Figures 2B-E).

We then applied auxin (indole-3-acetic acid, IAA) in lanoline paste directly to the axils of intact leaves in order to test if auxin can delay abscission of shaded leaves (Figure 3). Auxin application to the very proximal end of the petiole delayed darkinduced leaf abscission highly significantly by approximately 1 day. In order to examine if auxin can also work as a longdistance signal, which is transported from the leaf blade to the axil, we applied auxin (IAA) to the most distal end of the petiole. Also in this case, abscission was significantly delayed supporting the idea that auxin not only acts locally but has the potential to be a long-distance signal in leaf abscission.

\section{Inhibition of Polar Auxin Transport Delays Formation of Abscission Zones}

The polar auxin transport inhibitor morphactin (9hydroxyfluorene-9-carboxylic acid, CF) has previously been show to delay leaf abscission in citrus (Goren et al., 1986). Local application of morphactin to the axil of shaded leaves delayed separation by approximately 7 days, to an even stronger extent as observed for the application of the auxin influx carrier substrate 2,4-D (Figure 4A). We then tested whether morphactin modulates auxin response in leaf axils. In mocktreated axils, 6 days after shading started, a new local GH3::GUS maximum appeared at the lower side of the petiole. After 9 days, in partly separated petioles, most of the auxin response reporter activity occurred on the proximal side of the abscission zone (Figures 4B-D). By contrast, in presence of morphactin formation of the new auxin response maxium as well as of the abscission zone were delayed and only became visible 18 days after shading started (Figures 4E-G). Hence, morphactin likely acts on the formation of the abscission zone by preventing the establishment of a new auxin response maximum.

\section{Auxin Transporters are Down-Regulated during Abscission}

In order to identify auxin transporters, which could be involved in the formation of a new auxin response maximum during abscission we performed a microarray experiment. Total RNA was isolated from dissected leaf axils 9 days after shading started and from axils covered with transparent plastic bags. Among the approximately 2400 differentially expressed genes auxin related transcripts were strongly overrepresented (Supplemental Dataset). Within the group of the 200 most strongly downregulated genes several $A U X / I A A s, S A U R s$ but also the putative
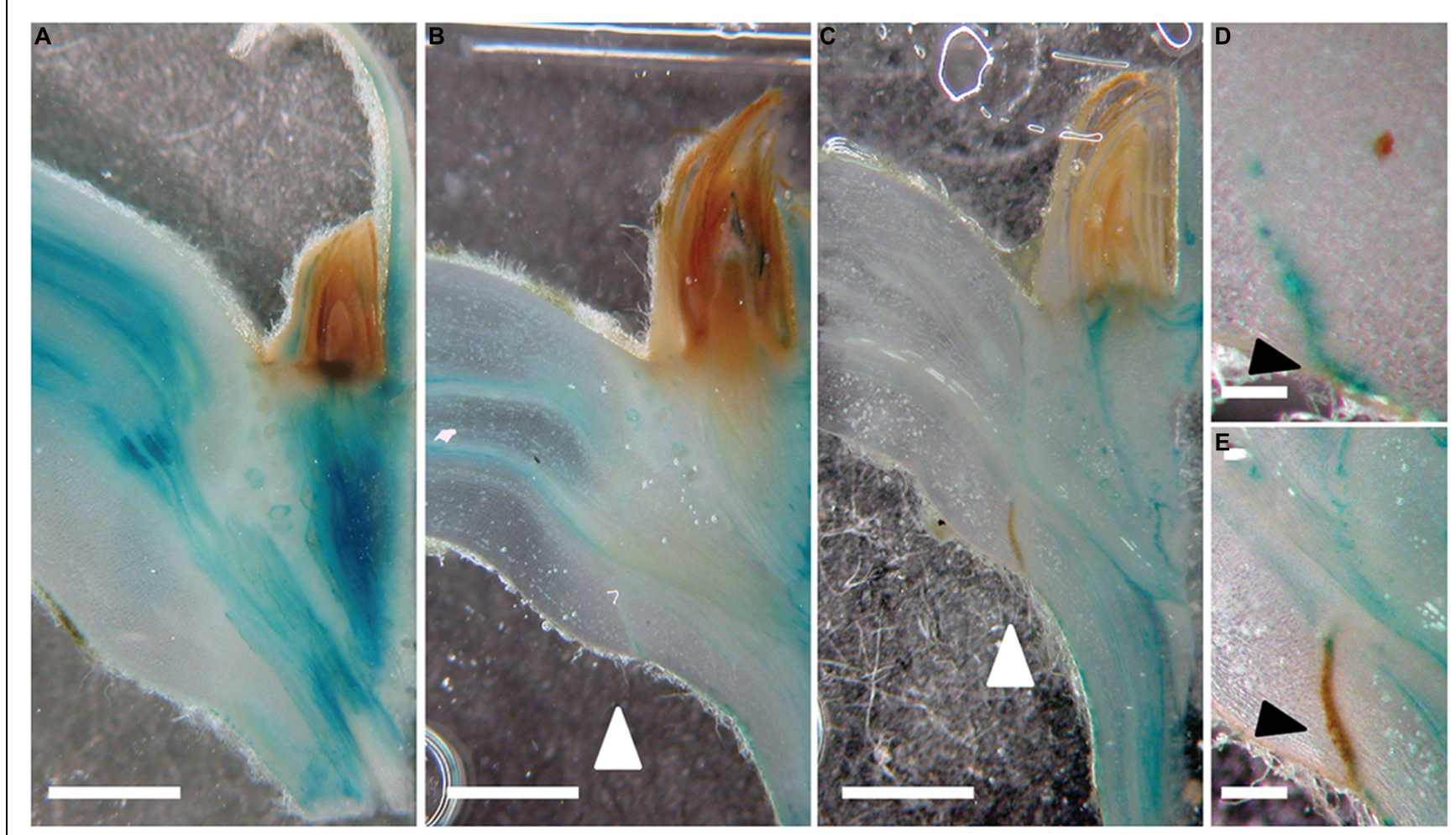

FIGURE 2 | A new auxin response maximum is established prior to the formation of an abscission zone. (A-E) GH3::GUS; 0 (A), 6 (B,D), and

9 days $(\mathbf{C}, \mathbf{E})$ after shading started. White arrow heads point to the abscission zones. (E) Mature abscission zone appears in brown; GUS precipitate in blue. Scale bars correspond to approximately $1 \mathrm{~mm}$ (A-C); 0,5 mm (D,E). Black arrowheads point to the forming (D) and mature abscission zone (E). 


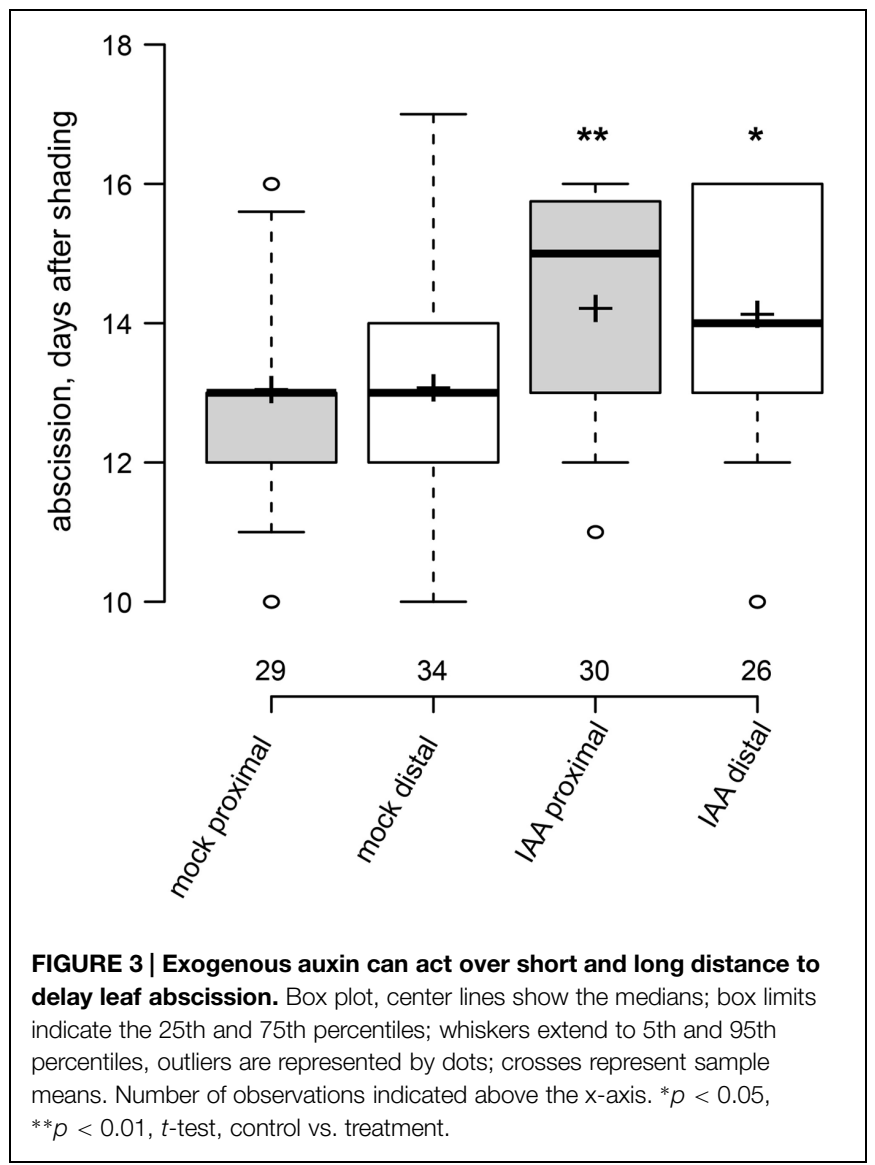

auxin transporters PtrPIN1b, PtrPIN5b, and PtrWAT1 were present (Table 1; data available at NCBI GEO, GSE69277). By contrast, none of the known putative auxin transporters were up-regulated. As its closest Arabidopsis homolog PIN1, PtrPIN1b localizes to the plasma membrane (Liu et al., 2014) and is most likely involved in intercellular auxin transport. On the other hand, the closest homologs of PtrPIN5b (PIN5) and PtrWAT1 (WAT1) localize in Arabidopsis to the endoplasmic reticulum and the tonoplast, respectively (Mravec et al., 2009; Ranocha et al., 2013), and are therefore expected to be involved in intracellular auxin transport and homeostasis. We isolated promoter fragments of PttPIN1b, PttPIN5b, PttWAT1, and used them to drive the GUS reporter gene. Expression of all reporter gene constructs was inducible by exogenous application of auxin (Supplementary Figure S1). In axils from non-shaded leaf blades, pPttPIN1b::GUS reporter activity was mainly associated with the vascular system across the entire axil (Figure 5A). Nine days after shading started additional pPttPIN1b::GUS activity was observed along the differentiating abscission zone (Figure 5D). Similarly, expression of the intracellular auxin transporters was most prominent in vascular tissues of axils from non-shaded leaf blades (Figures 5B,C). Shading led to strong, patchy expression of PtrPIN5b::GUS and PtrWAT1::GUS on the proximal side of the emerging abscission zone, whereas these genes were hardly expressed on the distal side (Figures 5E,F). These findings underline the importance of auxin transport in the formation

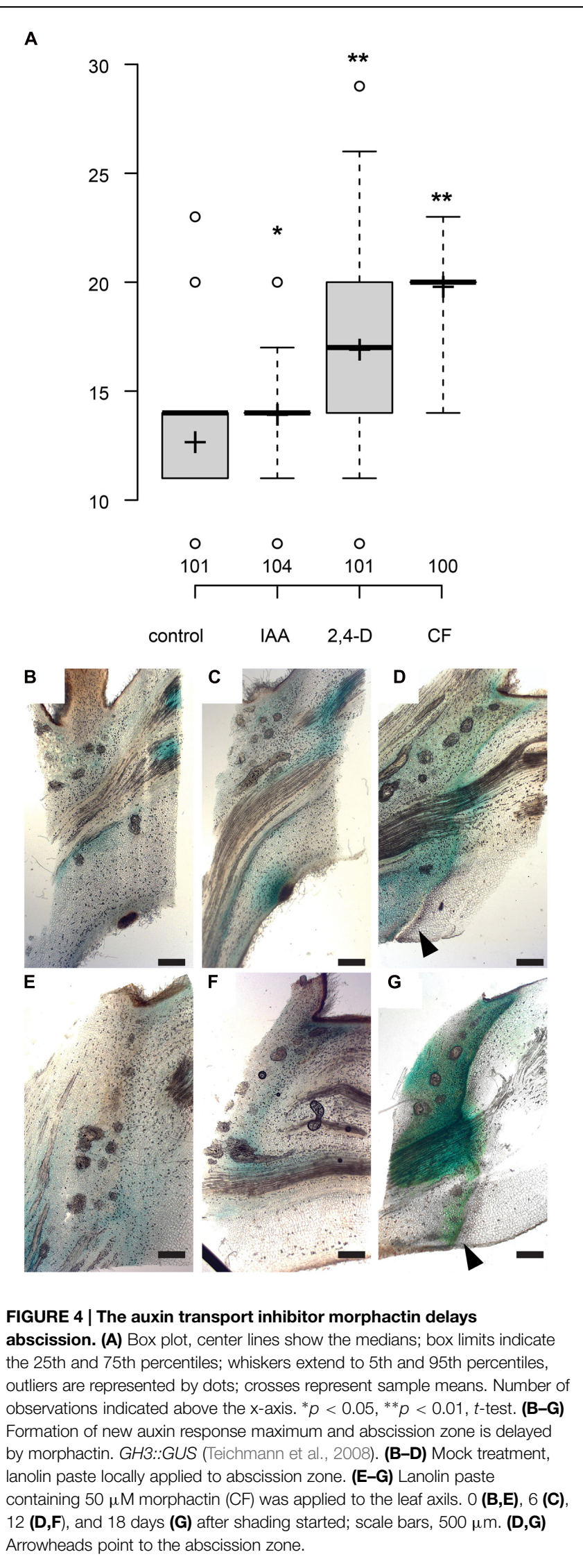


TABLE 1 | Genes involved in auxin transport and response are down-regulated in axils of shaded leaves.

\begin{tabular}{|c|c|c|c|c|c|c|}
\hline Rank & ID & $\log F C$ & $P$-value & adj.P-Value & $\begin{array}{l}\text { Closest At } \\
\text { homolog }\end{array}$ & Process \\
\hline 8 & PtpAffx.16882.2.S1_at & $-5,8$ & $6,7 \mathrm{E}-10$ & 4,6E-06 & IAA19 & AUX/IAA \\
\hline 19 & PtpAffx.25068.1.A1_at & $-4,7$ & $1,5 \mathrm{E}-07$ & $7,2 \mathrm{E}-05$ & IAA4 & AUX/IAA \\
\hline 20 & PtpAffx.7696.4.S1_a_at & $-4,7$ & $1,8 \mathrm{E}-08$ & 2,7E-05 & IAA4 & AUX/AA \\
\hline 21 & Ptp.128.1.S1_at & $-4,7$ & 2,7E-09 & 1,3E-05 & $\mid A A 14$ & AUX/IAA \\
\hline 30 & Ptp.6148.1.S1_at & $-4,4$ & $1,4 \mathrm{E}-06$ & 1,9E-04 & PIN5 & Efflux \\
\hline 31 & PtpAffx.7696.2.A1_a_at & $-4,4$ & 4,4E-07 & $1,1 \mathrm{E}-04$ & IAA4 & AUX/AA \\
\hline 36 & PtpAffx.213779.1.S1_at & $-4,3$ & 9,7E-08 & 5,9E-05 & SAUR75 & Response \\
\hline 40 & PtpAffx.21075.1.S1_at & $-4,2$ & 6,8E-07 & 1,3E-04 & SAUR14 & Response \\
\hline 45 & PtpAffx.73583.1.S1_at & $-4,0$ & 8,7E-08 & 5,7E-05 & IAA4 & AUXIAA \\
\hline 47 & PtpAffx.102281.1.A1_at & $-4,0$ & $2,7 \mathrm{E}-06$ & 2,7E-04 & SAUR75 & Respone \\
\hline 50 & Ptp.127.1.S1_s_at & $-4,0$ & 7,6E-07 & $1,4 \mathrm{E}-04$ & IAA4 & AUX/IAA \\
\hline 60 & Ptp.1099.1.A1_at & $-3,9$ & $7,1 \mathrm{E}-07$ & 1,4E-04 & GH3-10 & Homoestasis \\
\hline 74 & PtpAffx.7696.4.S1_at & $-3,6$ & 1,7E-07 & 7,8E-05 & IAA4 & AUX/IAA \\
\hline 75 & PtpAffx.204265.1.S1_at & $-3,6$ & $1,3 E-04$ & 3,0E-03 & SAUR14 & Response \\
\hline 84 & Ptp.1274.1.S1_s_at & $-3,5$ & $8,2 \mathrm{E}-06$ & $5,0 \mathrm{E}-04$ & PIN1 & Efflux \\
\hline 94 & PtpAffx.204337.1.S1_at & $-3,3$ & $1,5 \mathrm{E}-06$ & $2,0 \mathrm{E}-04$ & SAUR63 & Reponse \\
\hline 97 & PtpAffx.123174.1.A1_at & $-3,2$ & $2,1 \mathrm{E}-06$ & 2,3E-04 & IAA4 & AUX/IAA \\
\hline 103 & PtpAffx.21896.2.S1_s_at & $-3,2$ & 3,7E-07 & $1,1 \mathrm{E}-04$ & IAA13 & AUX/AA \\
\hline 113 & PtpAffx.249.95.S1_a_at & $-3,0$ & $1,2 \mathrm{E}-07$ & 6,6E-05 & WAT1 & Homeostasis \\
\hline 161 & Ptp.6738.1.S1_at & $-2,7$ & 2,2E-04 & 4,1E-03 & SAUR51 & Response \\
\hline 11 & Ptp.6116.1.S1_at & 4,5 & $1,1 \mathrm{E}-05$ & $5,8 \mathrm{E}-04$ & SUR2 & Homeostasis \\
\hline 13 & PtpAffx.54125.1.A1_s_at & 4,5 & 6,8E-06 & $4,5 \mathrm{E}-04$ & SUR2 & Homeostasis \\
\hline 94 & PtpAffx.210014.1.S1_at & 3,1 & $1,8 \mathrm{E}-04$ & 3,6E-03 & GH3-6 & Homeostasis \\
\hline 180 & PtpAffx.221307.1.S1_at & 2,6 & 4,0E-05 & 1,3E-03 & SAUR17 & Response \\
\hline
\end{tabular}

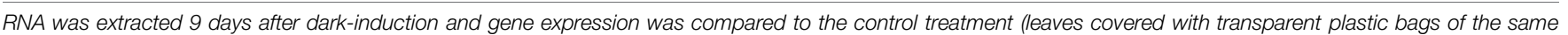

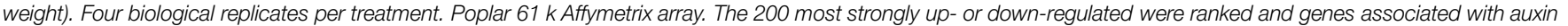
transport, response, or homeostasis were extracted from this list. Rank 1 corresponds to the most strongly up-and down-regulated genes, respectively.

of a new auxin maximum and the formation of an abscission zone.

\section{Regulation of Auxin Transport during Abscission is Independent of Ethylene Signaling}

We then wondered if auxin acts through ethylene signaling on leaf abscission as it has been suggested previously (for review, Estornell et al., 2013). We first analyzed abscission in 35S::etr1-1 ethylene insensitive trees (Love et al., 2009). Expression of the dominant Arabidopsis etr1-1 allele under the control of the $35 \mathrm{~S}$ promoter renders Populus trees insensitive to ethylene. 35S:: etr1-1 trees formed anatomically normally looking abscission zones after shading of leaf blades indicating that early steps of abscission could take place in absence of ethylene signaling (Figures 6A,B). 35S::etr1-1 leaves, however, separated later or not at all from the plant body (Figure 6C). We then tested if the expression of auxin transporter genes is reduced after shading in 35S::etr1-1 trees to a similar extent than in wild type. In wild-type leaf axils, PttPIN1b, PttPIN5b, and PttWAT1 were significantly lower 6 days after shading started compared to non-shaded leaf axils (Figure 6D). Similar levels of dark-induced reduction in expression of PttPIN1b and PttPIN5b were observed in the ethylene-insensitive 35S::etr1-1 trees (Figure 6D). By contrast, close homologs of the pectin methylesterase QUARTET1 and the polygalacturonase $A D P G 1$, which are required for cell separation during microgenesis and dehiscence (Francis et al., 2006; Ogawa et al., 2009), were among the most strongly upregulated genes after leaf shading and their up-regulation was dependent on ethylene signaling (Figure 6E; Supplementary Table S2). Together these findings suggest that the regulation of auxin transporters and the formation of an abscission zone are independent of ethylene signaling. In order to test if auxin acts on leaf abscission solely upstream of ethylene or in an independent parallel pathway we tested if auxin has an effect on the timing of abscission in 35S::etr1-1 axils of shaded leaves (Figure 6C). Exogenous auxin further delayed abscission and the number of leaves, which did not abscise, in an additive manner, as compared to mock treated 35S::etr1-1 axils, suggesting that auxin acts in an independent pathway, parallel to ethylene signaling, on leaf abscission.

\section{Discussion}

Based on earlier work, an auxin gradient spanning the abscission zone has been proposed to regulate timing of organ separation (Addicott et al., 1955). Instead of an auxin response gradient we observed a discrete auxin response maximum, which precedes the 


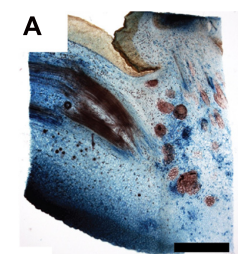

B
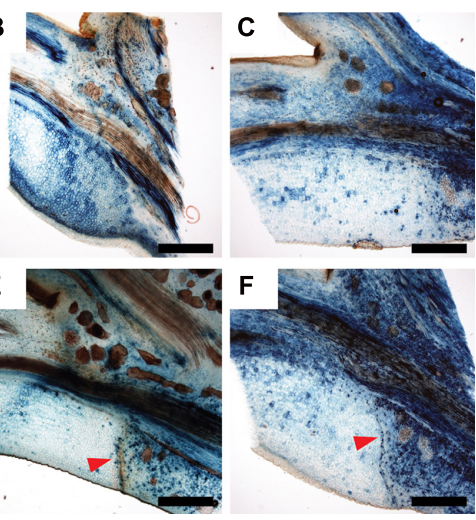

FIGURE 5| Expression of WAT1::GUS and PIN5b::GUS is shifted to the proximal side of the abscission zone after shading of leaf blades. (A,D) PtrPIN1b::GUS; (B,E) PtrWAT1::GUS; (C,F) PtrPIN5b::GUS. (A-C) axils of non-shaded leaf blades; (D-F) 9 days after shading started. Red arrowheads point to mature abscission zone. Scale bars, $1 \mathrm{~mm}$.

formation of an abscission zone, indicating that auxin provides positional cues for the specification and/or differentiation of an abscission zone. Similar spatially discrete auxin maxima or gradients have been proposed to regulate the positioning of leaf and lateral root primordia as well as the zonation patterning in the cambium (Dubrovsky et al., 2008; Kierzkowski et al., 2013; Bhalerao and Fischer, 2014). In the cambium high auxin concentrations correlate with meristematic activity and cell division of protoplasts requires the presence of auxin (Bhalerao and Fischer, 2014). Intriguingly, formation of the abscission zone not only involves specialization of cells but also in many cases cell proliferation. In contrast to an auxin response maximum, which precedes the formation of an abscission zone, Sorefan et al. (2009) reported that a local auxin response minimum is required for differentiation of the separation layer of the valve margin. At the site of reduced auxin response secondary walls are deposited, which are instrumental during the separation process. Interestingly, a strong auxin response is detected in the replum, the tissue between valve margins, coincidentally, overlapping with the region where cell divisions occur during the formation of an abscission zone (Wu et al., 2006; Sorefan et al., 2009). Thus, the mode of auxin action might be similar in abscission zones as suggested for the cambium, high concentrations permit cell division, below a certain threshold cell expansion and differentiation are favored.

Recently, an auxin response maximum has been observed in the abscission zone of Arabidopsis petals (Basu et al., 2013). In auxin influx carrier mutants, petal break strength is increased and therefore $A U X 1$ and its paralogs might be involved in the formation of an auxin concentration maximum in the abscission zone (Basu et al., 2013). In mutants of the auxin efflux carrier PIN4, additional root cap layers are observed (Friml et al., 2002). In wild-type, the outermost tier of the root cap is continuously shed from the root. The pin 4 phenotype is either a consequence of over-proliferation of root cap cells or, more likely, of impaired cell separation. Our findings of delayed auxin maximum formation and abscission upon inhibition of polar auxin transport and the strong reduction of auxin carriers in dark-induced leaf axils are in support with the idea that auxin transport is instrumental for the establishment of an instructive auxin response maximum during abscission.

Interestingly, expression of iaaL, which conjugates auxin, under the control of an abscission zone specific polygalacturonase promoter results in reduced auxin response and increased petal break strength (Basu et al., 2013). Polygalacturonases are integral parts of cell wall remodeling during the separation process (Roongsattham et al., 2012) and it is likely that the promoter used to drive iaaL is specifically active during later phases of abscission rather than during the formation of an abscission zone. Consequently, relatively low auxin concentrations during the separation phase are sufficient to hasten abscission. Hence, auxin is likely to have a dual function during abscission in first providing positional information for the formation of the abscission zone and secondly as a signal, which regulates temporal aspects of the separation. Such a mode-of-action ensures that under high auxin concentrations an abscission zone can differentiate but separation cannot be initiated. As a consequence premature abscission, which can be deleterious for plant performance, might be prevented.

Ethylene induced reduction of auxin transport capacity in the midrib of poplar leaves correlates with leaf abscission (Riov and Goren, 1979). Furthermore, it has been suggested that reduced uptake of auxin into the cells of the abscission zone increases their sensitivity toward ethylene (Taylor and Whitelaw, 2001). We observed that formation of an abscission zone and the regulation of auxin transport in petioles of dark-induced leaves are independent of ethylene signaling. Importantly, auxin could further delay abscission of ethylene insensitive petioles. Therefore, auxin may act in parallel and independently of ethylene on the hydrolysis of middle lamellae. The existence of such an ethylene-independent, additional signaling pathway has been suggested earlier, based on the observation that petal abscission in the ethylene insensitive mutants ein2 and etr1-1 is only incompletely inhibited (Bleecker and Patterson, 1997).

Evidence for a non-cell-automonous signal, which provides positional information for the formation of an abscission zone, was first provided by the analyses of the MADS box transcription factor JOINTLESS in tomato. jointless mutants do not form abscission zones in pedicels (Mao et al., 2000). Interestingly, JOINTLESS function in in the clonal layer L3 (vasculature) is sufficient to rescue the jointless phenotype. This finding strongly suggests that JOINTLESS can act through a mobile factor on cells in the cortex and epidermis of the pedicel to induce the formation of an abscission zone (Szymkowiak and Irish, 1999). The establishment of a new auxin maximum prior to the maturation of the abscission zone and regulation of auxin transporters in the abscission zone after dark induction are arguments in favor of auxin to provide cues to position the abscission zone, similar as for the formation of primordia in apical meristems and during differentiation of cambial derivatives. Intriguingly, exogenous auxin is sufficient to induce ectopic expression of the peptide hormone INFLORESCENCE DEFICIENT IN ABSCISSION (IDA), which controls cell separation during floral 


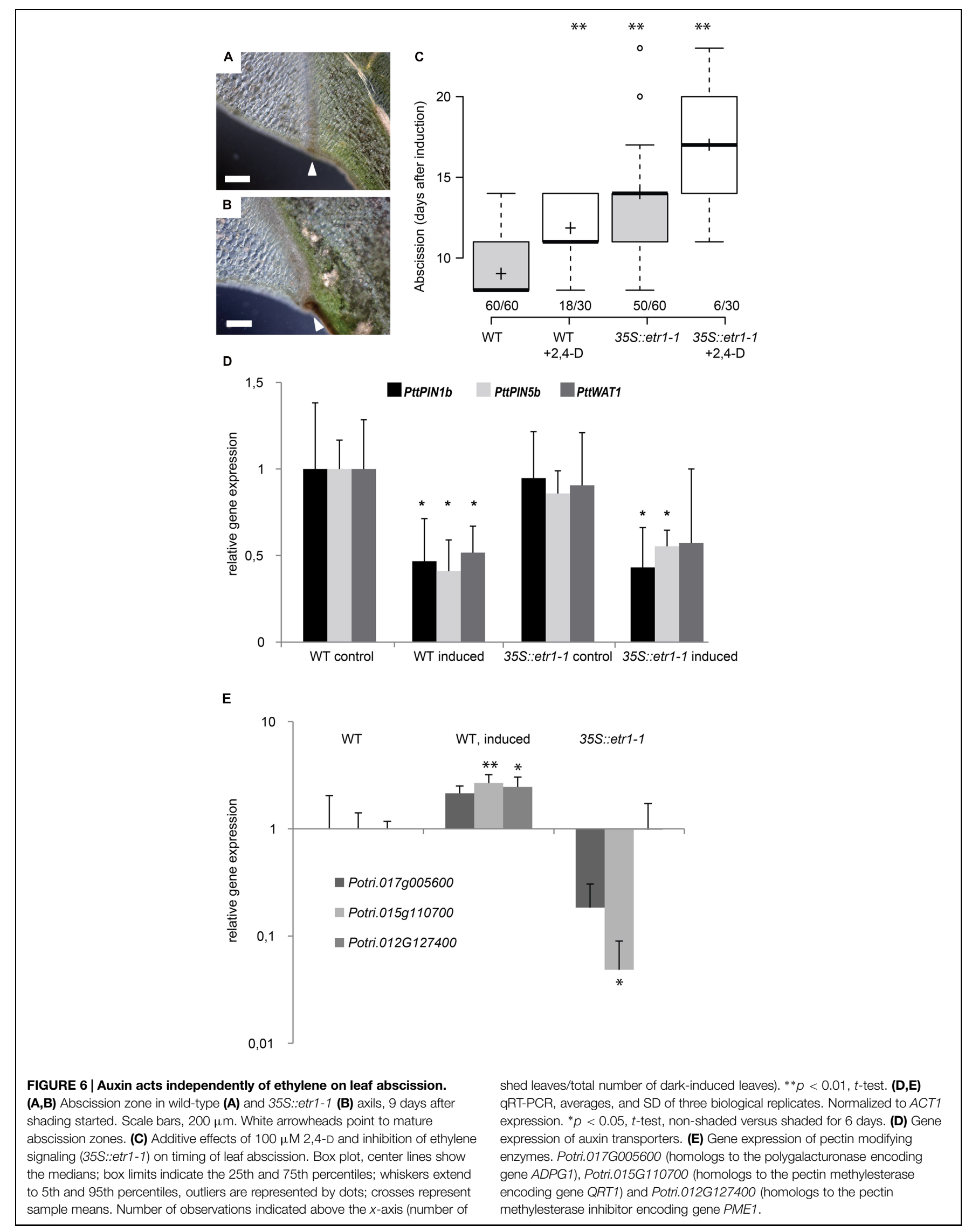


organ abscission and lateral root primordia emergence (Aalen et al., 2013; Kumpf et al., 2013). Besides the functional analyses of the identified auxin transporters PtrPIN1b, PtrPIN5b and PtrWAT1, future directions of research should include the determination of subcellular localization of auxin efflux carriers in the petiole. Subsequent modeling of auxin distribution could reveal if a scenario of auxin providing positional information to the cells in the petiole is plausible.

\section{Author contributions}

$\mathrm{XJ}$, JZ, and UF performed and analyzed the experiments. AP and UF designed the research and edited the manuscript. XJ and UF wrote the manuscript.

\section{References}

Aalen, R. B., Wildhagen, M., Sto, I. M., and Butenko, M. A. (2013). IDA: a peptide ligand regulating cell separation processes in Arabidopsis. J. Exp. Bot. 64, 5253-5261. doi: 10.1093/jxb/ert338

Addicott, F. T., and Lynch, R. S. (1955). Physiology of abscission. Annu. Rev. Plant Phys. 6, 211-238. doi: 10.1146/annurev.pp.06.060155.001235

Addicott, F. T., Lynch, R. S., and Carns, H. R. (1955). Auxin gradient theory of abscission regulation. Science 121, 644-645. doi: 10.1126/science.121.3148.644

Aponte, C., Garcia, L. V., and Maranon, T. (2013). Tree species effects on nutrient cycling and soil biota: a feedback mechanism favouring species coexistence. For. Ecol. Manag. 309, 36-46. doi: 10.1016/j.foreco.2013.05.035

Basu, M. M., Gonzalez-Carranza, Z. H., Azam-Ali, S., Tang, S., Shahid, A. A., and Roberts, J. A. (2013). The manipulation of auxin in the abscission zone cells of Arabidopsis flowers reveals that indoleacetic acid signaling is a prerequisite for organ shedding. Plant Physiol. 162, 96-106. doi: 10.1104/pp.113.216234

Bhalerao, R. P., and Fischer, U. (2014). Auxin gradients across wood-instructive or incidental? Physiol. Plantarum. 151, 43-51. doi: 10.1111/ppl.12134

Bleecker, A. B., and Patterson, S. E. (1997). Last exit: senescence, abscission, and meristem arrest in Arabidopsis. Plant Cell 9, 1169-1179. doi: 10.1105/tpc.9.7.1169

Chen, S. L., Wang, S. S., Altman, A., and Huttermann, A. (1997). Genotypic variation in drought tolerance of poplar in relation to abscisic acid. Tree Physiol. 17, 797-803. doi: 10.1093/treephys/17.12.797

Dubrovsky, J. G., Sauer, M., Napsucialy-Mendivil, S., Ivanchenko, M. G., Friml, J., Shishkova, S., et al. (2008). Auxin acts as a local morphogenetic trigger to specify lateral root founder cells. Proc. Natl Acad. Sci. U.S.A. 105, 8790-8794. doi: 10.1073/pnas.0712307105

Ellis, C. M., Nagpal, P., Young, J. C., Hagen, G., Guilfoyle, T. J., and Reed, J. W. (2005). AUXIN RESPONSE FACTOR1 and AUXIN RESPONSE FACTOR2 regulate senescence and floral organ abscission in Arabidopsis thaliana. Development 132, 4563-4574. doi: 10.1242/dev.02012

Estornell, L. H., Agusti, J., Merelo, P., Talon, M., and Tadeo, F. R. (2013). Elucidating mechanisms underlying organ abscission. Plant Sci. 199, 48-60. doi: 10.1016/j.plantsci.2012.10.008

Fischer, U., and Polle, A. (2010). "Populus responses to abiotic stress," in Genetics and Genomics of Populus, Vol. 8, eds S. Jansson, R. P. Bhalerao, and A. Groover (New York, NY: Springer), 225-246. doi: 10.1007/978-1-4419-15 41-2_11

Francis, K. E., Lam, S. Y., and Copenhaver, G. P. (2006). Separation of Arabidopsis pollen tetrads is regulated by QUARTET1, a pectin methylesterase gene. Plant Physiol. 142, 1004-1013. doi: 10.1104/pp.106.085274

Friml, J., Benkova, E., Blilou, I., Wisniewska, J., Hamann, T., Ljung, K., et al. (2002). AtPIN4 mediates sink-driven auxin gradients and root patterning in Arabidopsis. Cell 108, 661-673. doi: 10.1016/S0092-8674(02)00656-6

Giovannelli, A., Deslauriers, A., Fragnelli, G., Scaletti, L., Castro, G., Rossi, S., et al. (2007). Evaluation of drought response of two poplar clones (Populus $\mathrm{x}$ canadensis Monch 'I-214' and P-deltoides Marsh. 'Dvina') through high

\section{Acknowledgments}

This work was supported by Deutsche Forschungsgemeinschaft DFG (Fi1661/1-1 to UF), Bio4Energy, the Berzelii Centre and Stiftelsen Mauritz Carlgrens Fond (XJ). We would like to thank Rishikesh P. Bhalerao for helping to improve the manuscript; Christine Kettner, Benedikt Wingen, Hardy Hall, Daniela Liebsch, Weiya Xue, Qian Ma, Huizu Guo, and Tao Yang for technical assistance.

\section{Supplementary Material}

The Supplementary Material for this article can be found online at: http://journal.frontiersin.org/article/10.3389/fpls.2015.00634

resolution analysis of stem growth. J. Exp. Bot. 58, 2673-2683. doi: 10.1093/jxb/erm117

Goren, R., Aron, Y., Monselise, S. P., and Huberman, M. (1986). Morphactininduced inhibition of abscission in citrus leaves. Acta Hortic. 179, 631-637.

Hong, S. B., Sexton, R., and Tucker, M. L. (2000). Analysis of gene promoters for two tomato polygalacturonases expressed in abscission zones and the stigma. Plant Physiol. 123, 869-881. doi: 10.1104/pp.123.3.869

Jiang, C.-Z., Lu, F., Imsabai, W., Meir, S., and Reid, M. S. (2008). Silencing polygalacturonase expression inhibits tomato petiole abscission. J. Exp. Bot. 59, 973-979. doi: 10.1093/jxb/ern023

Keskitalo, J., Bergquist, G., Gardestrom, P., and Jansson, S. (2005). A cellular timetable of autumn senescence. Plant Physiol. 139, 1635-1648. doi: 10.1104/pp.105.066845

Kierzkowski, D., Lenhard, M., Smith, R., and Kuhlemeier, C. (2013). Interaction between meristem tissue layers controls phyllotaxis. Dev. Cell. 26, 616-628. doi: 10.1016/j.devcel.2013.08.017

Kumpf, R. P., Shi, C.-L., Larrieu, A., Sto, I. M., Butenko, M. A., Peret, B., et al. (2013). Floral organ abscission peptide IDA and its HAE/HSL2 receptors control cell separation during lateral root emergence. Proc. Natl Acad. Sci. U.S.A. 110, 5235-5240. doi: 10.1073/pnas.1210835110

La Rue, C. D. (1936). The effect of auxin on the abscission of petioles. Proc. Natl Acad. Sci. U.S.A. 22, 254-259. doi: 10.1073/pnas.22.5.254

Lewis, M. W., Leslie, M. E., and Liljegren, S. J. (2006). Plant separation: 50 ways to leave your mother. Curr. Opin. Plant Biol. 9, 59-65. doi: 10.1016/j.pbi.2005.11.009

Liu, B., Zhang, J., Wang, L., Li, J., Zheng, H., Chen, J., et al. (2014). A survey of Populus PIN-FORMED family genes reveals their diversified expression patterns. J. Exp. Bot. 65, 2437-2448. doi: 10.1093/jxb/eru129

Lohse, M., Nunes-Nesi, A., Kruger, P., Nagel, A., Hannemann, J., Giorgi, F. M., et al. (2010). Robin: an intuitive wizard application for R-Based expression microarray quality assessment and analysis. Plant Physiol. 153, 642-651. doi: $10.1104 /$ pp.109.152553

Louie, D. S., and Addicott, F. T. (1970). Applied auxin gradients and abscission in explants. Plant Physiol. 45, 654-657. doi: 10.1104/pp.45.6.654

Love, J., Bjorklund, S., Vahala, J., Hertzberg, M., Kangasjarvi, J., and Sundberg, B. (2009). Ethylene is an endogenous stimulator of cell division in the cambial meristem of Populus. Proc. Natl Acad. Sci. U.S.A. 106, 5984-5989. doi: 10.1073/pnas.0811660106

Mao, L., Begum, D., Chuang, H. W., Budiman, M. A., Szymkowiak, E. J., Irish, E. E., et al. (2000). JOINTLESS is a MADS-box gene controlling tomato flower abscission zone development. Nature 406, 910-913. doi: 10.1038/35022611

Mravec, J., Skupa, P., Bailly, A., Hoyerova, K., Krecek, P., Bielach, A., et al. (2009). Subcellular homeostasis of phytohormone auxin is mediated by the ERlocalized PIN5 transporter. Nature 459, 1136-1140. doi: 10.1038/nature08066

Nilsson, O., Alden, T., Sitbon, F., Little, C. H. A., Chalupa, V., Sandberg, G., et al. (1992). Spatial pattern of cauliflower mosaic virus 35S promoter-luciferase expression in transgenic hybrid aspen trees monitored by enzymatic assay and non-destructive imaging. Transgenic Res. 1, 209-220. doi: 10.1007/BF02524751 
Ogawa, M., Kay, P., Wilson, S., and Swain, S. M. (2009). ARABIDOPSIS DEHISCENCE ZONE POLYGALACTURONASE1 (ADPG1), ADPG2, and QUARTET2 are Polygalacturonases Required for Cell Separation during reproductive development in Arabidopsis. Plant Cell 21, 216-233. doi: 10.1105/tpc.108.063768

Olsen, J. E., Junttila, O., Nilsen, J., Eriksson, M. E., Martinussen, I., and Olsson, O. (1997). Ectopic expression of oat phytochrome a in hybrid aspen changes critical daylength for growth and prevents cold acclimatization. Plant J. 12, 1339-1350. doi: 10.1046/j.1365-313x.1997.12061339.x

Patterson, S. E. (2001). Cutting loose. Abscission and dehiscence in Arabidopsis. Plant Physiol. 126, 494-500. doi: 10.1104/pp.126.2.494

Patterson, S. E., and Bleecker, A. B. (2004). Ethylene-dependent and -independent processes associated with floral organ abscission in Arabidopsis. Plant Physiol. 134, 194-203. doi: 10.1104/pp.103.028027

Ranocha, P., Dima, O., Nagy, R., Felten, J., Corratge-Faillie, C., Novak, O., et al. (2013). Arabidopsis WAT1 is a vacuolar auxin transport facilitator required for auxin homoeostasis. Nat. Commun. 4:2625. doi: 10.1038/ncomms3625

Riov, J., and Goren, R. (1979). Effect of ethylene on auxin transport and metabolism in midrib sections in relation of leaf abscission of woody plants. Plant Cell Environ. 2, 83-89. doi: 10.1111/j.1365-3040.1979.tb00778.x

Roberts, J. A., Elliott, K. A., and Gonzalez-Carranza, Z. H. (2002). Abscission, dehiscence, and other cell separation processes. Ann. Rev. Plant Biol. 53, 131-158. doi: 10.1146/annurev.arplant.53.092701.180236

Roongsattham, P., Morcillo, F., Jantasuriyarat, C., Pizot, M., Moussu, S., Jayaweera, D., et al. (2012). Temporal and spatial expression of polygalacturonase gene family members reveals divergent regulation during fleshy fruit ripening and abscission in the monocot species oil palm. BMC Plant Biol. 12:150. doi: 10.1186/1471-2229-12-150

Sorefan, K., Girin, T., Liljegren, S. J., Ljung, K., Robles, P., Galvan-Ampudia, C. S., et al. (2009). A regulated auxin minimum is required for seed dispersal in Arabidopsis. Nature 459, 583-586. doi: 10.1038/nature07875

Street, N. R., Skogstrom, O., Sjodin, A., Tucker, J., Rodriguez-Acosta, M., Nilsson, P., et al. (2006). The genetics and genomics of the drought response in Populus. Plant J. 48, 321-341. doi: 10.1111/j.1365-313X.2006. 02864.x

Szymkowiak, E. J., and Irish, E. E. (1999). Interactions between jointless and wildtype tomato tissues during development of the pedicel abscission zone and the inflorescence meristem. Plant Cell 11, 159-175. doi: 10.1105/tpc.11.2.159

Taylor, J. E., and Whitelaw, C. A. (2001). Signals in abscission. New Phytol. 151, 323-339. doi: 10.1046/j.0028-646x.2001.00194.x

Teichmann, T., Bolu-Arianto, W. H., Olbrich, A., Langenfeld-Heyser, R., Goebel, C., Grzeganek, P., et al. (2008). GH3: GUS reflects cell-specific developmental patterns and stress-induced changes in wood anatomy in the poplar stem. Tree Physiol. 28, 1305-1315. doi: 10.1093/treephys/28. 9.1305

Vahala, J., Felten, J., Love, J., Gorzsás, A., Gerber, L., Lamminmäki, A., et al. (2013). A genome-wide screen for ethylene-induced Ethylene Response Factors (ERFs) in hybrid aspen stem identifies ERF genes that modify stem growth and wood properties. New Phytol. 200, 511-522. doi: 10.1111/nph.12386

Wu, H., Mori, A., Jiang, X., Wang, Y., and Yang, M. (2006). The INDEHISCENT protein regulates unequal cell divisions in Arabidopsis fruit. Planta 224, 971979. doi: 10.1007/s00425-006-0351-8

Zanne, A. E., Tank, D. C., Cornwell, W. K., Eastman, J. M., Smith, S. A., FitzJohn, R. G., et al. (2014). Three keys to the radiation of angiosperms into freezing environments. Nature 506, 89-92. doi: 10.1038/nature 12872

Conflict of Interest Statement: The authors declare that the research was conducted in the absence of any commercial or financial relationships that could be construed as a potential conflict of interest.

Copyright (c) 2015 Jin, Zimmermann, Polle and Fischer. This is an open-access article distributed under the terms of the Creative Commons Attribution License (CC BY). The use, distribution or reproduction in other forums is permitted, provided the original author(s) or licensor are credited and that the original publication in this journal is cited, in accordance with accepted academic practice. No use, distribution or reproduction is permitted which does not comply with these terms. 